\title{
A FEC Decoding Iterative Algorithm for DVB-S2 in Variable Coding and Modulation System
}

\author{
Han $\mathrm{Hao}^{1, \text { a }}$,Jinshu Chen ${ }^{1, \mathrm{~b}}$ and Ying Zhou ${ }^{2, \mathrm{c}}$ \\ ${ }^{1}$ Department of Electronic Engineering, 100084 Tsinghua University, Beijing, China \\ ${ }^{2}$ Department of Civil Engineering, 100084 Tsinghua University, Beijing, China \\ ahaohan14@mails.tsinghua.edu.cn, bchenjs@mails.tsinghua.edu.cn, \\ 'zhouying16@mails.tsinghua.edu.cn
}

\begin{abstract}
Keywords:DVB-S2;VCM; FEC decoding iterative algorithm
Abstract.A decoding iterative algorithm for second generation digital video broadcasting (DVB-S2) standard forward error correction (FEC) and variable coding and modulation (VCM) is proposed in this paper. This method makes full use of the link budget information provided by the VCM system. It predicts the number of decoding iterations for different modes, reduces the average number of iterations by $62 \%$, and does not increase the checking cost. Then, using the FEC decoding structure of DVB-S2, the fast and reliable convergence of decoding is completed without increasing the complexity.
\end{abstract}

\section{Introduction}

DVB-S2 is a set of standards standardized by the DVB organization and adopted by the International Telecommunication Union (ITU) and the European Telecommunications Standards Institute (ETSI) as the official standard, and is widely used in the field of satellite communications due to its excellent performance. Its performance advantage mainly comes from $\mathrm{BCH}$ cascaded LDPC forward error correction (FEC) codec system. Meanwhile, DVB-S2 supports 4 modulation mapping methods and 11 LDPC coding rates. Through reasonable configuration and combination, it is possible to realize a VCM system which can select different coding and modulation (C\&M) modes according to different channel conditions, so as to obtain higher data transmission efficiency[1].

The main problem of DVB-S2 system FEC decoding is how efficient implementation of LDPC decoding. A conventional method of LDPC decoding is iterative decoding. Because of the long code length of DVB-S2 standard LDPC code, the normal frame length is 64800 bits and the short frame length is 16200 bits, thedecoding process is complicated. Each iteration consumes considerable latency and power consumption, it is necessary to precisely control the number of iterations. In general, LDPC decoding uses the parity check matrix of the output codeword to determine whether to continue iterations [2]. This algorithm to stop the iteration must meet a very stringent condition, that is completely correct decoding. Based on this method, a series of iterative algorithm to relax the requirement for stopping iteration are proposed [3,4], but inevitably at the expense of performance degradation. Meanwhile, for DVB-S2 system, this kind of method will increase the consumption of memory resource or the time delay and calculation amount brought by complicated route addressing and checking calculation. Another widely used algorithm of decoding iteration is based on the method of studying the trend of extrinsic information [5,6,7]. This method can effectively control the average number of iterations.But the disadvantage is that the decoder structure must be changed, thus increasing the computational complexity.A practical alternative is to set a fixed number of iterations [8], which has the advantage of being simple to implement.However, in order to guarantee the decoding performance, it needs some redundant iterations, and the number of iterations is imprecise. In addition, $\mathrm{C}$. Marchand et al. Proposed a novel idea to reduce the average number of iterations by using the FEC outer code error correction capability for the DVB-X2 $(X=S, C, T)$ series of standards [9], but the decision method is more complex. 
On the other hand, the data transmission efficiency of VCM system is significantly better than Constant Coding and Modulation (CCM) system [10].At the same time, VCM is simpler than Adaptive Coding and Modulation (ACM) system and does not need to interact with the satellite via real-time remote control signals. Therefore, VCM is very suitable for earth observation satellite data reception and other application scenarios.However, there are few studies on FEC decoding iterative methods for VCM.

\section{DVB-S2 Standard VCM Profile}

In CCM system, C\&Mmode is fixed.Therefore, the link budget must meet the worst channel conditions.However, in actual communication missions, channel conditions tend to be dynamic [11].Taking the receiving mission of Earth observation satellites as an example, considering the link loss change with the change of elevation angle, the fluctuation is as high as $14 \mathrm{~dB}$ [12].

VCM system presets the C\&M variation scheme according to the channel conditions and the link budget before the communication mission. The track is divided into a fixed number of sectors (such as 6), to avoid the overhead and system performance degradation generated by excessive C\&M mode switching [10]. In the case of good channel conditions(taking into account factors such as weather) or sectors, a high-order modulation and high-rate coding mode shall be selected to obtain higher data transfer rate. On the contrary, select the low-order modulation and low-rate coding mode to ensure the system transmission performance. A mode set including $28 \mathrm{C} \& \mathrm{M}$ modes is given as a CandidateofVCM system. And the theoretical SNR of each model to meet the performance requirements, called IDEAL SNR, is given by MATLAB simulation [1].

Compared with CCM, the data throughput of VCM increased by 35.5\% [12]. The system structure of VCM is shown in Fig. 1 and Fig. 2, where FEC codec are in red boxes, which are the concerns of this paper.

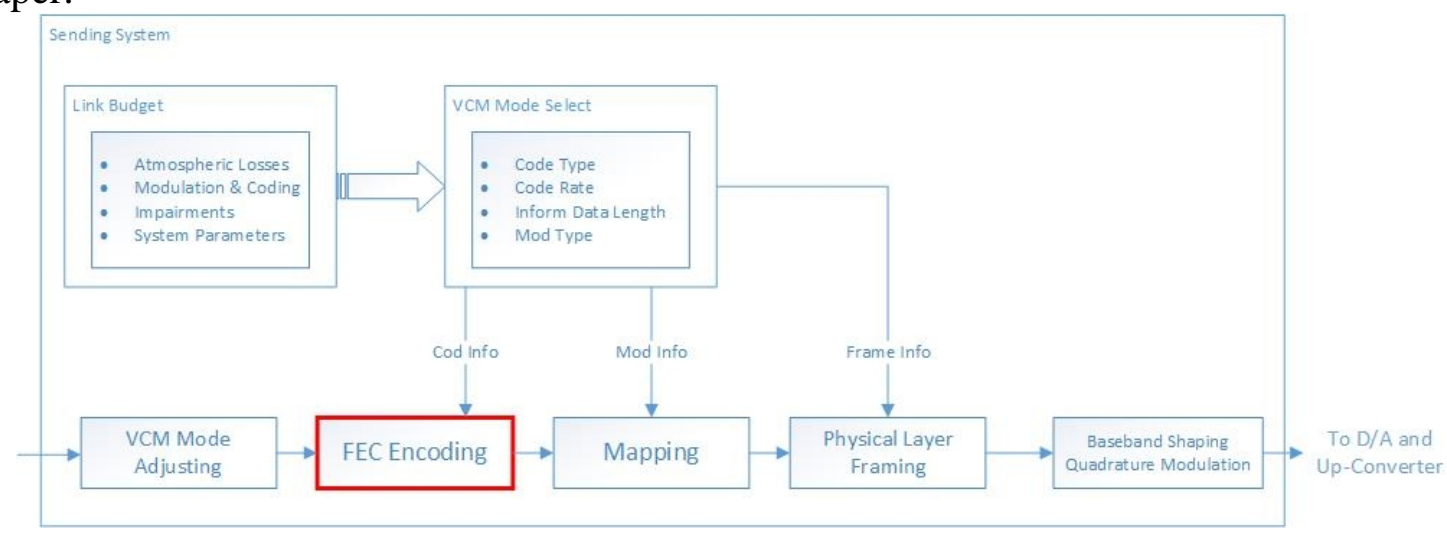

Fig. 1VCM system transmitter structure

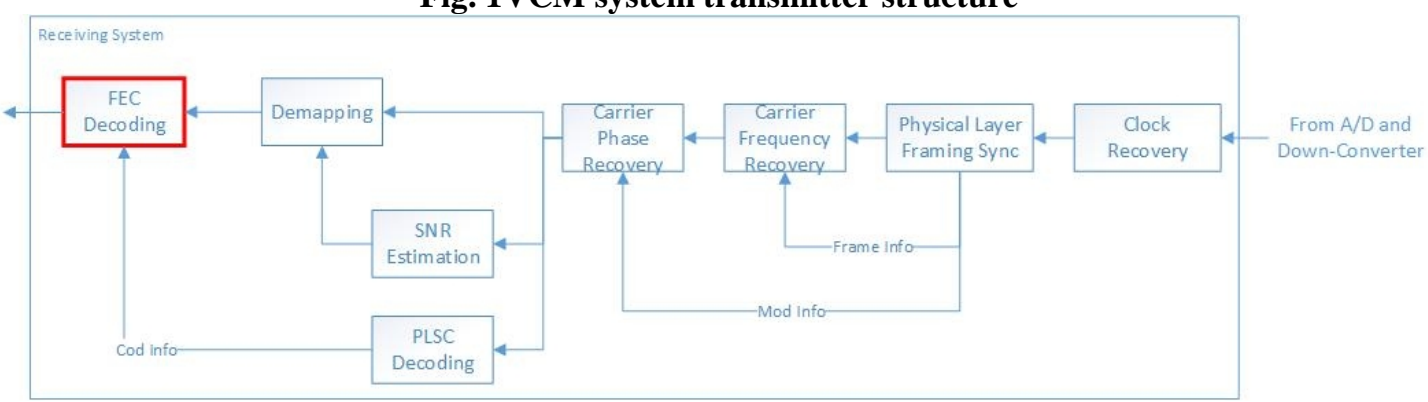

Fig. 2VCM system receiver structure

\section{Analysis of DVB - S2 FEC Encoding and Decoding}

DVB-S2 FEC codec structure.DVB-S2 FEC using BCH code (outer code) and LDPC code (inner code) cascade structure [1].Each $\mathrm{K}_{b c h}$ bits input stream (BBFRAME) is subjected to BCH encoding 
and LDPC encoding in succession to generate an $n_{l d p c}$ bits output stream (FECFRAME). As shown in Fig. 3, parity bits (BCHFEC \& LDPCFEC) of the outer code and the inner code are sequentially connected after BBFRAME to form a system code. When the FECFRAME bit interleaving is finished, the FEC encoding is completed.

\begin{tabular}{|c|c|c|}
\hline \multicolumn{2}{|c|}{$\mathbf{N}_{b c h}=\mathbf{k}_{l d p c}$} & \multirow[b]{2}{*}{$\mathrm{n}_{l d p c}-\mathrm{k}_{l d p}$} \\
\hline $\mathrm{K}_{b c h}$ & $\mathrm{~N}_{b c h}-\mathrm{K}_{b c h}$ & \\
\hline BBFRAME & BCHFEC & LDPCFEC \\
\hline \multicolumn{3}{|c|}{$\mathrm{n}_{l d p c}$ bits } \\
\hline
\end{tabular}

Fig. 3 FECFRAME structure

The FEC decoding process is the inverse of encoding process.An $n_{l d p c}$ bits FECFRAME obtained by deinterleaving a frame of demapping data is subjected to LDPC and BCH decoding to output $\mathrm{K}_{b c h}$ bits BBFRAME in order.

The DVB-S2 standard specifies the methods of interleaving and deinterleaving, the parameters of FEC encoding and decoding, and the coding method. So this paper will not discuss the above issues. The following describes the FEC decoding algorithm, including $\mathrm{BCH}$ code decoding algorithm and LDPC code decoding algorithm.

BCH decoding algorithm.In this paper, $\mathrm{BCH}$ decoding algorithm based on $\mathrm{BM}$ algorithm is adopted [13].The algorithm steps are as follows:

Step1: Calculate the syndrome $\mathrm{S}$ from the received codeword polynomial $\mathrm{R}(\mathrm{x})$.

Let the received codeword polynomial be $\mathrm{R}(x)=r_{n-1} x^{n-1}+r_{n-2} x^{n-2}+\cdots+r_{1} x+r_{0}$. Syndrome $S=\left(s_{1}, s_{2}, s_{3}, \ldots, s_{2 t}\right)$. And $s_{i}=R\left(\alpha^{i}\right), i=1,2,3, \ldots, 2 t . \alpha^{i}$ is the root of $2 \mathrm{t}$ successive powers of $\mathrm{BCH}$ code generator polynomial, which can be obtained by look-up table. $t$ is the number of errors that the BCH code can correct, as defined by the DVB-S2 standard [1].

Step 2: Using the BM algorithm, calculate the error position polynomial $\sigma(x)$ by syndrome $\mathrm{S}$.

i. Initialization.

$$
\sigma^{(-1)}(x)=1, \sigma^{(0)}(x)=1, d_{-1}=1, D(-1)=0
$$

ii. Calculate correction as Eq. 2

$$
d_{j}=s_{j+1}+\sum_{i=1}^{\partial^{\circ} \sigma^{(j)}(x)} s_{j+1-i} \sigma_{i}^{(j)}
$$

$\partial^{\circ} \sigma^{(j)}(x)$ denotes the highest power in $\sigma^{(j)}(x) . \sigma_{i}^{(j)}$ is the coefficient of $x^{i}$ in $\sigma^{(j)}(x)$. If $d_{j}=0$, then $\sigma^{(j+1)}(x)=\sigma^{(j)}(x)$. Otherwise, denotes $\mathrm{D}(i)=\partial^{\circ} \sigma^{(i)}(x)$ and find a row $i$ before $j$, making $i-$ $\mathrm{D}(i)$ value to the maximum and $\mathrm{d}_{i}$ is not equal to zero. Then, calculate

$$
\sigma^{(j+1)}(x)=\sigma^{(j)}(x)-d_{j} d_{i}^{-1} x^{j-i} \sigma^{(i)}(x)
$$

iii. Calculate $d_{j+1}$ and repeat ii step to iterate. After 2 t iterations, $\sigma^{2 t}(x)$ is obtained. While $\mathrm{D}(2 t)$ is the number of error bits for $\mathrm{BCH}$ error detection.

Step 3: Use Chien search to find the root of $\sigma(x)=0$ and correct the errors.

The $\mathrm{t}$ roots of $\sigma(x)=0$ must be in $\left(\alpha^{k}\right)^{-1}, k=0,1,2, \ldots, n-1$. Check whether $\left(\alpha^{k}\right)^{-1}, k=$ $0,1,2, \ldots, n-1$ is root one by one. The corresponding $\mathrm{k}$ is the error location. Invert this bit to correct the error for binary $\mathrm{BCH}$.

LDPC decoding algorithm [14].Firstly let's give some definition: $y_{v}$ denotes the initialized data of variable node $\mathrm{v}$ after passing through the channel. $I_{v}$ denotes the channel intrinsic information Log-Likelihood Ratio (LLR) of variable node v. $L_{v}^{k}$ is the posterior message of variable node $\mathrm{v}$ in the $k^{t h}$ iteration. $R_{C \rightarrow v}^{k}$ is the extrinsic message of check node c passed to variable node $\mathrm{v}$ in the $k^{t h}$ 
iteration. $L_{v \rightarrow c}^{k}$ is the prior message of variable node $\mathrm{v}$ passed to check node $\mathrm{c}$ in the $k^{\text {th }}$ iteration. $N_{(c)}$ denotes a set of all variable nodes associated with check node c. "/" is the symbol of exclusion. $\operatorname{sgn}(x)$ is the symbol of computing the sign of $\mathrm{x} . x_{v}^{k}$ is the hard-decision value of variable node $\mathrm{v}$ in the $k^{\text {th }}$ iteration. IteraConstrepresents the constant number of iterations.

In this paper, TDMP-based min-sum decoding algorithm is adopted. The algorithm steps are as follows:

Step 1: Initialization.

$$
L_{v}^{0}=I_{v}=-\frac{2 y_{v}}{\sigma^{2}}, R_{c \rightarrow v}^{0}=0
$$

Step 2: Update the extrinsic message of check nodes in the update layer.

i. Prior message is updated first.

$$
L_{v \rightarrow c}^{k}=L_{v}^{k-1}-R_{c \rightarrow v}^{k-1}(5)
$$

ii. Update the extrinsic message of check nodes in the layer with the updated prior message.

$$
R_{c \rightarrow v}^{k}=\prod_{n \in N_{(c)} \backslash v} \operatorname{sgn}\left(L_{n \rightarrow c}^{k}\right) \times \min _{n \in N_{(c)} \backslash v}\left|L_{n \rightarrow c}^{k}\right|(6)
$$

Step 3: Update the variable node posterior messages associated with check nodes in the layer.

$$
L_{v}^{k}=L_{v \rightarrow c}^{k}+R_{c \rightarrow v}^{k}(7)
$$

Step 4: Repeat step 2 and 3 till all layer has been processed. One iteration is complete.

Step 5: Hard decision.

$$
x_{v}^{k}=\left\{\begin{array}{ll}
1 & L_{v}^{k}<0 \\
0 & L_{v}^{k} \geq 0
\end{array}(8)\right.
$$

Step 6: Set a fixed number of iterationsIteraConst. Ifk $\geq$ IteraConst, stop the iteration and output $x_{v}^{k}$. Otherwise, repeat step 2 to 6.

\section{Proposed FEC decoding iteration algorithm and simulation results}

A. Variable iteration algorithm based on VCM. Conventional constant iteration algorithm is actually to guarantee the decoding performance under the worst channel condition. However, the channel condition of communication mission is roughly predicted and divided by the VCM system. Considering this factor, MATLAB simulation analysis is carried out for the maximum number of LDPC decoding iterations under different VCM modes and IDEAL SNRs in AWGN channel. The simulation results are shown in Fig. 4. Limited by simulation conditions, simulation decoding performance is 0 error in 5E7 bits.

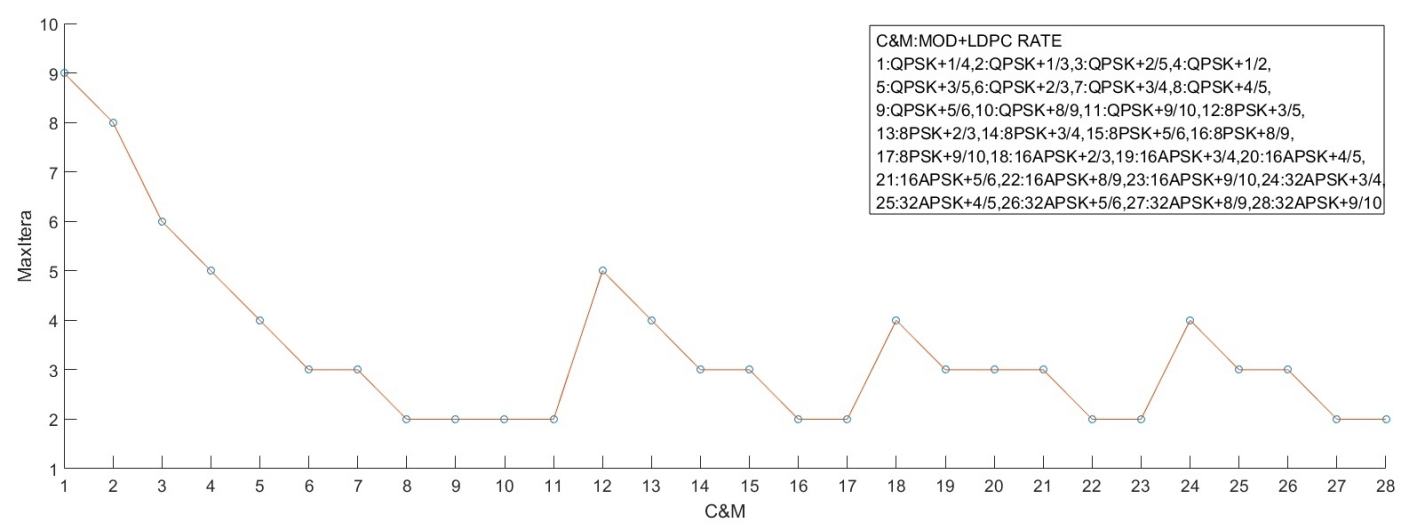

Fig. 4Maximum number of LDPC decoding iterations for VCM modes

It can be seen from Fig. 4 that if we use the conventional constant iteration algorithm, each FECFRAME requires 9 LDPC decoding iterations. Considering the characteristics of VCM, a new 
variable iteration algorithm based on VCM is proposed. The number of LDPC decoding iterations of each FECFRAME is determined according to the C\&M mode information known before decoding. At the same complexity as conventional constant iteration algorithm, the minimum number of LDPC decoding iterations required per frame is only 2 . If the probability of each channel condition is equal, the average number of LDPC decoding iterations required for decoding each FECFRAME is 3.43 times, down by $62 \%$.

B. Optimization: Multi-threshold-based FEC hybrid decoding iterative algorithm. The advantage of variable iteration algorithm is the simple implementation and less resource consumption. However, it lacks verification of the decoding results and completely based on the simulation results. Considering that the FEC decoding structure of DVB-S2 is LDPC and BCH concatenation. BCH code has an error detection and correction capability of not more than t, can verify the LDPC decoding result without adding any resources. Meanwhile, by using this feature of $\mathrm{BCH}$, we can relax the stopping condition of LDPC decoding iteration from the number of error bits per frame equal to 0 to not more than $\mathrm{t}$. Thereby further reducing the number of LDPC decoding iterations [9]. Therefore, a multi-threshold-based FEC hybrid decoding iterative algorithm is derived. Flow diagram shown in Fig. 5. The number of iterations denoted as "Itera", and the number of error bits as "NOE"."IteraUL" means the upper limit of iteration.

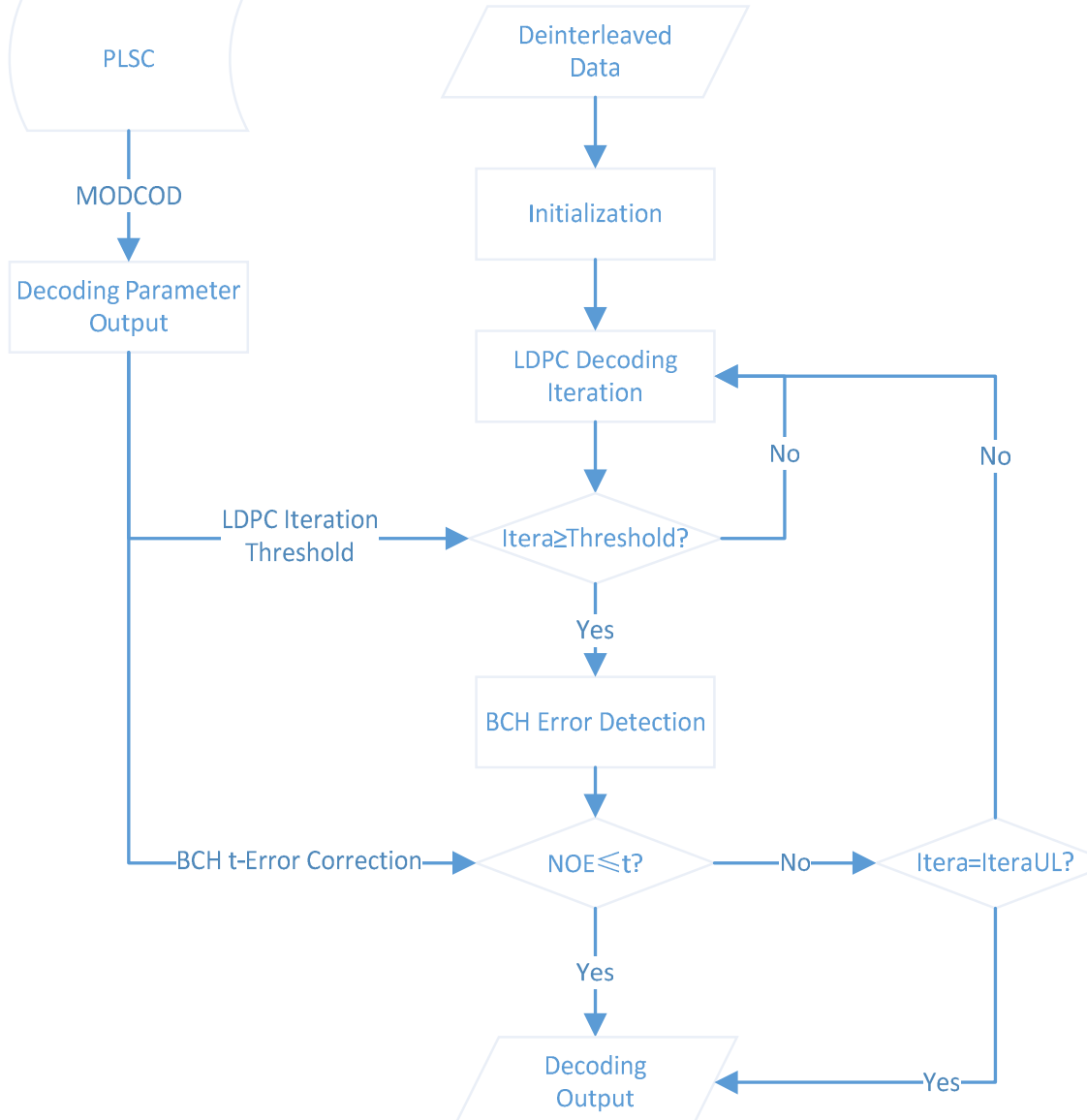

Fig. 5Flow diagram of multi-threshold-based FEC hybrid decoding iterative algorithm

C. Threshold selecting in multi-threshold-based algorithm. Based on the algorithm described in A, it is clear that the maximum number of iterations in the lowest SNR of the current mode should be used to ensure decoding performance. However, after the optimization in $\mathrm{B}$, it is necessary to consider the balance between LDPC decoding iteration and $\mathrm{BCH}$ error detection.

Some conclusions can be drawn from Fig. 5. Threshold selection is too low, will increase the number of $\mathrm{BCH}$ error detection. On the contrary, it will increase the unnecessary LDPC decoding iterations. 
Take the normal frame QPSK + 2/3 LDPC mode as an example. The complexity of one BCH error detection and one LDPC decoding iteration are shown in Table 1.

Table 1Comparison of complexity between BCH error detection and LDPC decoding iteration

\begin{tabular}{|c|c|c|c|c|c|}
\hline \multirow{2}{*}{$\begin{array}{c}\text { BCH or } \\
\text { LDPC }\end{array}$} & Addition & Subtraction & Comparison & XOR & And \\
\cline { 2 - 6 } LDPC & $4.9 \mathrm{E} 6$ & $7.3 \mathrm{E} 5$ & $3.4 \mathrm{E} 6$ & $4.5 \mathrm{E} 5$ & 0 \\
\hline BCH & $1.5 \mathrm{E} 2$ & $1.6 \mathrm{E} 2$ & $8.6 \mathrm{E} 5$ & $1.4 \mathrm{E} 7$ & $1.3 \mathrm{E} 2$ \\
\hline
\end{tabular}

It can be seen that the computational complexity of one $\mathrm{BCH}$ error detection or one LDPC decoding iteration is equivalent. Therefore, it is not advisable to exchange one LDPC decoding iteration with multiple $\mathrm{BCH}$ error detection. $\mathrm{BCH}$ error detection, however, contains a large number of XOR operations is relatively more simple. Therefore, it should be selected between equal number of $\mathrm{BCH}$ error detection and LDPC decoding iterations.

In the iterative decoding of one FECFRAME, there is always a minimum positive integer called optimal number of LDPC iterations, denoted as $L I^{O P}$. So that the number of error bits after $L I^{O P}$ LDPC iterations is not more than $\mathrm{t}$, and then the correct output can be achieved after one $\mathrm{BCH}$ error detection and decoding.

Suppose there are k possible optimal numbers of LDPC iterations $L I_{1}^{O P}, L I_{2}^{O P}, \ldots, L I_{k}^{O P}$ in the SNR interval (denoted as $\left[S N R_{\min }, S N R_{\max }\right]$ ) of a certain mode. The optimal probability density in $\left[S N R_{\min }, S N R_{\max }\right]$ are $f_{1}(x), f_{2}(x), \ldots, f_{k}(x)$ respectively. Obviously, the optimal number of $\mathrm{BCH}$ error detection is always 1 .

The threshold is assumed to be $L I_{x}$. When the optimal number of LDPC iterations is $L I_{i}^{O P}$ ( $i \in$ $1,2, \ldots, \mathrm{k})$, the actual number of LDPC iterations is

$$
L I_{i}^{A V}=\left\{\begin{array}{l}
L I_{i}^{O P} L I_{x}<L I_{i}^{O P} \\
L I_{x} L I_{x} \geq L I_{i}^{O P}
\end{array}\right.
$$

And the actual number of $\mathrm{BCH}$ error detection is

$$
B I_{i}^{A V}=\left\{\begin{array}{cl}
L I_{i}^{O P}-L I_{x}+1 & L I_{x}<L I_{i}^{O P} \\
1 & L I_{x} \geq L I_{i}^{O P}
\end{array}\right.
$$

Calculate the total number of LDPC iteration and BCH error detection as Eq. 11

$$
S I_{i}^{A V}=L I_{i}^{A V}+B I_{i}^{A V}=\left\{\begin{array}{cc}
2 L I_{i}^{O P}-L I_{x}+1 & L I_{x}<L I_{i}^{O P} \\
L I_{x}+1 & L I_{x} \geq L I_{i}^{O P}
\end{array}\right.
$$

The number of additional iterations or detection is

$$
S I_{i}^{E X}=S I_{i}^{A V}-L I_{i}^{O P}-1=\left|L I_{x}-L I_{i}^{O P}\right|(12)
$$

Consider the average number of additional iterations or detection in $\left[S N R_{\min }, S N R_{\max }\right]$

$$
A I^{E X}=\sum_{i=1}^{k} \int_{S N R_{\min }}^{S N R_{\max }}\left|L I_{x}-L I_{i}^{O P}\right| * f_{i}(x) d x, i \in 1,2, \ldots, k(13
$$

For any fixed $i, L I_{x}$ and $L I_{i}^{O P}$ are determined values. Therefore

$$
A I^{E X}=\sum_{i=1}^{k}\left|L I_{x}-L I_{i}^{O P}\right| * \int_{S N R_{\min }}^{S N R_{\max }} f_{i}(x) d x, i \in 1,2, \ldots, k(14
$$

Select $L I_{x}$ as the threshold from $L I_{i}^{O P}(i \in 1,2, \ldots, \mathrm{k})$ to minimize the $A I^{E X}$ value. The complexity of $\mathrm{BCH}$ error detection and LDPC decoding iteration shall be in consideration, thus the smallest $L I_{i}^{O P}$ can be selected when there are multiple $A I^{E X}$ values of $L I_{i}^{O P}$ close.

D. Recommended threshold and performance comparison. According to the discussion of $\mathrm{C}$, set the threshold for each mode as shown in Table 2 . 
Table 2Threshold for different mode of VCM

\begin{tabular}{|c|c|c|c|c|c|c|c|}
\hline C\&M No. & Threshold & C\&M No. & Threshold & C\&M No. & Threshold & C\&M No. & Threshold \\
\hline 1 & 3 & 2 & 3 & 3 & 2 & 4 & 2 \\
\hline 5 & 2 & 6 & 1 & 7 & 1 & 8 & 1 \\
\hline 9 & 1 & 10 & 1 & 11 & 1 & 12 & 2 \\
\hline 13 & 2 & 14 & 2 & 15 & 1 & 16 & 1 \\
\hline 17 & 1 & 18 & 2 & 19 & 2 & 20 & 1 \\
\hline 21 & 1 & 22 & 1 & 23 & 1 & 24 & 1 \\
\hline 25 & 1 & 26 & 1 & 27 & 1 & 28 & 1 \\
\hline \multicolumn{8}{|c|}{$\begin{array}{l}\text { C\&M No.: Modulation + LDPC rate } \\
\text { 1:QPSK+1/4,2:QPSK+1/3,3:QPSK+2/5,4:QPSK+1/2,5:QPSK+3/5,6:QPSK+2/3,7:QPSK+3/4, } \\
\text { 8:QPSK+4/5,9:QPSK+5/6,10:QPSK+8/9,11:QPSK+9/10,12:8PSK+3/5,13:8PSK+2/3, } \\
\text { 14:8PSK+3/4,15:8PSK+5/6,16:8PSK+8/9,17:8PSK+9/10,18:16APSK+2/3,19:16APSK+3/4, } \\
\text { 20:16APSK+4/5,21:16APSK+5/6,22:16APSK+8/9,23:16APSK+9/10,24:32APSK+3/4, } \\
\text { 25:32APSK+4/5,26:32APSK+5/6,27:32APSK+8/9,28:32APSK+9/10 }\end{array}$} \\
\hline
\end{tabular}

Experiments are conducted to investigate performance of the proposed algorithm. Fig. 6 shows that to achieve the same decoding performance ( 0 errors in 5E7 bits) in the AWGN channel, the average numbers of LDPC decoding iterations required for algorithm $\mathrm{A}$ and $\mathrm{B}$. And the average numbers of $\mathrm{BCH}$ error detection can be drawn in Fig 7. Algorithm $\mathrm{A}$ in figures is multi-threshold-based FEC hybrid decoding iterative algorithm, and B is conventional constant iteration algorithm.

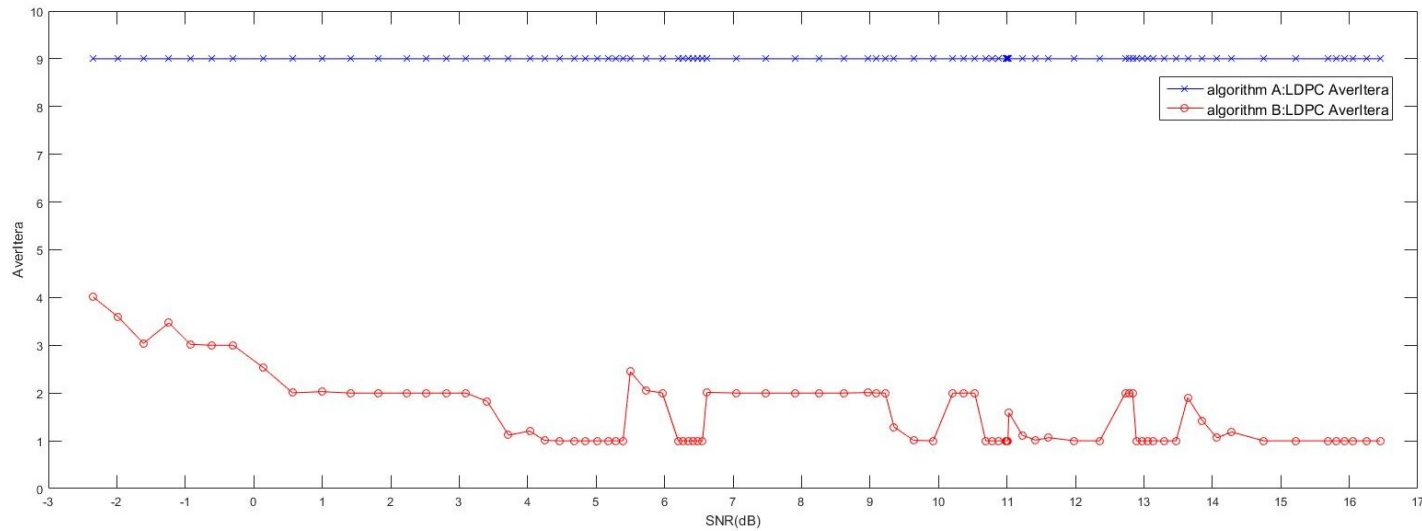

Fig. 6Comparison of average LDPC iterations

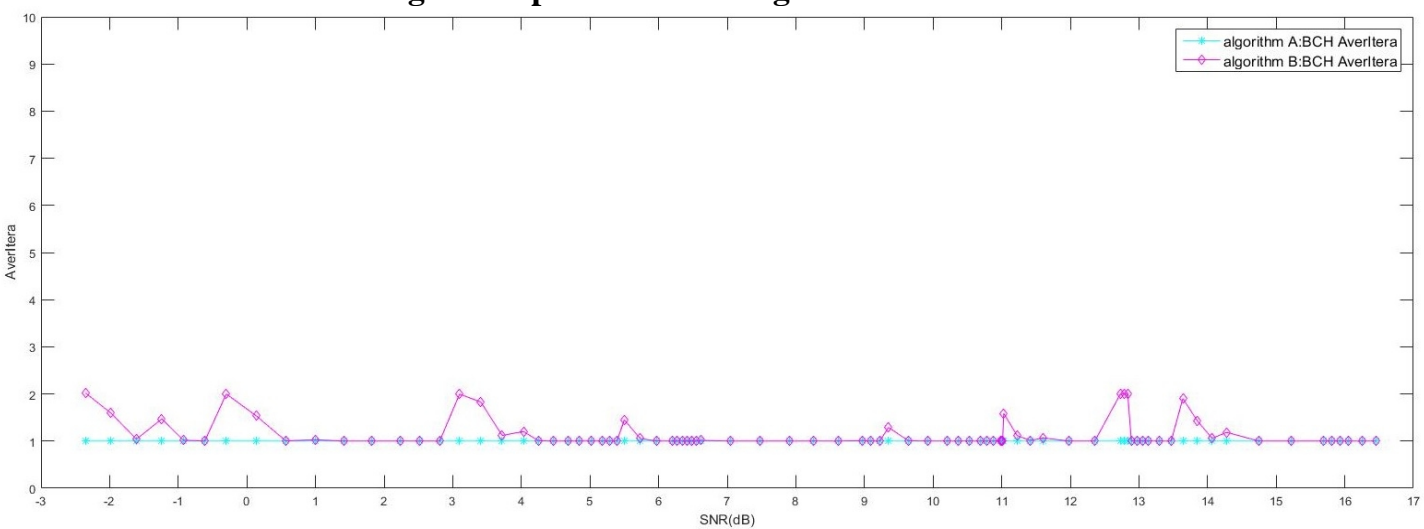

Fig. 7Comparison of average BCH error detection

It can be seen that the proposed decodingiterativealgorithm greatly reduces the number of LDPC decoding iterations by increasing a small amount of $\mathrm{BCH}$ error detection. In fact, this performance comes from a combination of several factors. Firstly, iterative thresholds set for different VCM modes make iteration more accurate. Second, $\mathrm{BCH}$ error detection provides the ability to verify LDPC decoding results, eliminating the blind redundancy of LDPC iterations. Thirdly, the LDPC iterative stopping condition is relaxed by using the error correction capability of $\mathrm{BCH}$. 


\section{Conclusions}

In this paper, we study the FEC decoding characteristics of DVB-S2 standard VCM system, and propose a multi-threshold-based FEC hybrid decoding iterative algorithm. Compared with the conventional constant iterative decoding algorithm, the proposed algorithm has the same resource consumption and significantly reduces the number of LDPC decoding iterations by exploiting the channel conditions implied by the VCM C\&M mode and the inherent LDPC and BCH cascade structure of DVB-S2. The cost is only a small increase in the $\mathrm{BCH}$ error detection, which is simpler than LDPC iteration. Benefit from this, decoding delay and power consumption will be reduced.

\section{References}

[1] Digital Video Broadcasting (DVB)-Second generation framing structure, channel coding and modulation systems for Broadcasting, Interactive Services, News Gathering and other broadband satellite applications, ETSI EN 302 307(2014).

[2] R. Gallager: IEEE Trans. Inf. TheoryVol. 8 (1962), p. 21-28.

[3] B. Shin, S. Kin, J. No and D. Shin: Proc. ISITA(2008), p. 1-5.

[4] G. Han and X. Liu: IEEE Commun Vol. 14 No. 11(2010), p. 1053-1055.

[5] F. Kienle and N. Wehn:Proc. IEEE Veh. Technol. Conf(2005), p. 606-609.

[6] J. Li, X. You, and J. Li: IEEE Commun. Lett. Vol. 10 No. 9(2006), p. 667-669.

[7] J. Hagenauer, E. Offer and L. Papke: IEEE Trans. Inf. Theory Vol. 42(1996), p. 429-449.

[8] J. S. Zhang and L. T. Guo: Video Engineering Vol. 36 No. 19(2012), p. 4-7In Chinese.

[9] C. Marchand and E. Boutillon: Electronics Letters Vol. 51 No. 1(2015), p. 114-116.

[10] M. Bertinelli and N. Toptsidis:VCM performance for LEO satellites at $26 \mathrm{GHz}$ a realistic assessment(ESA/Estec, 2010).

[11] T. Sielicki, J. Hamkins and D. Thorsen: submitted to IEEE Aerospace Conference (2013).

[12] X. Zhang: Telecommunication Engineering Vol. 54 No. 1(2014), p. 12-16 In Chinese.

[13] L. Fang: Design and Implementation of BCH Codec in DVB-S2 (2007) In Chinese.

[14] C. S. Zhou, Y. L. Ge, X. B. Chen, Y. Chen and X. Y. Zeng: Proc. ASAP (2011), p. 105-112. 